\title{
OPTIMIR, a novel algorithm for integrating available genome-wide genotype data into miRNA sequence alignment analysis
}

\author{
FLORIAN THIBORD, ${ }^{1,2,3}$ CLAIRE PERRET, ${ }^{1,2}$ MAGUELONNE ROUX, ${ }^{1,2}$ PIERRE SUCHON, ${ }^{4,5}$ \\ MARINE GERMAIN, ${ }^{1,2,3}$ JEAN-FRANÇOIS DELEUZE, ${ }^{6,7}$ PIERRE-EMMANUEL MORANGE, ${ }^{4,5}$ \\ and DAVID-ALEXANDRE TRÉGOUËT, ${ }^{1,2,3}$ on behalf of the GENMED CONSORTIUM \\ ${ }^{1}$ Sorbonne Universités, Université Pierre et Marie Curie (UPMC Univ Paris 06), Institut National pour la Santé et la Recherche Médicale \\ (INSERM), Unité Mixte de Recherche en Santé (UMR_S) 1166, Team Genomics and Pathophysiology of Cardiovascular Diseases, \\ 75013 Paris, France \\ ${ }^{2}$ Institute for Cardiometabolism and Nutrition (ICAN), 75013 Paris, France \\ ${ }^{3}$ INSERM UMR_S 1219, Bordeaux Population Health Research Center, University of Bordeaux, 33076 Bordeaux, France \\ ${ }^{4}$ Laboratory of Haematology, La Timone Hospital, 13885 Marseille, France \\ ${ }^{5}$ Institut National pour la Santé et la Recherche Médicale (INSERM), Unité Mixte de Recherche en Santé (UMR_S) 1062, Nutrition Obesity and Risk \\ of Thrombosis, Center for CardioVascular and Nutrition Research (C2VN), Aix-Marseille University, 13885 Marseille, France \\ ${ }^{6}$ Centre National de Recherche en Génomique Humaine, Direction de la Recherche Fondamentale, CEA, 91057 Evry, France \\ ${ }^{7} \mathrm{CEPH}$, Fondation Jean Dausset, 75011 Paris, France
}

\begin{abstract}
Next-generation sequencing is an increasingly popular and efficient approach to characterize the full set of microRNAs (miRNAs) present in human biosamples. MiRNAs' detection and quantification still remain a challenge as they can undergo different posttranscriptional modifications and might harbor genetic variations (polymiRs) that may impact on the alignment step. We present a novel algorithm, OPTIMIR, that incorporates biological knowledge on miRNA editing and genome-wide genotype data available in the processed samples to improve alignment accuracy. OPTIMIR was applied to 391 human plasma samples that had been typed with genome-wide genotyping arrays. OPTIMIR was able to detect genotyping errors, suggested the existence of novel miRNAs and highlighted the allelic imbalance expression of polymiRs in heterozygous carriers. OPTIMIR is written in python, and freely available on the GENMED website (http://www.genmed.fr/ index.php/fr/) and on Github (github.com/FlorianThibord/OptimiR).
\end{abstract}

Keywords: microRNA; next-generation sequencing; alignment; genetic variations; isomiRs

\section{INTRODUCTION}

With an average length of 22 nucleotides ( $\mathrm{nt}$ ), microRNAs (miRNAs) belong to a class of small noncoding RNAs known to regulate gene expression by binding messenger RNAs (mRNAs) and interfering with the translational machinery (Bartel 2004; Filipowicz et al. 2008). MiRNAs are transcribed from primary miRNA sequences (pri-miRNAs) and fold into a hairpin-like structure, which is sequentially processed by two ribonucleases, DROSHA and DICER. The former cleaves the pri-miRNA into a pre-miRNA and the latter completes the miRNA's maturation by cleaving the pre-miRNA near its loop to produce a miRNA duplex

Corresponding author: david-alexandre.tregouet@inserm.fr Article is online at http://www.rnajournal.org/cgi/doi/10.1261/rna. 069708.118. Freely available online through the RNA Open Access option. composed of two mature strands (Kim et al. 2009). Exceptionally, some miRNAs follow a slightly different pathway where only one ribonuclease is needed to complete the maturation (Kim et al. 2016). In any case, only one of the two mature strands is loaded in an effective protein complex called RISC, while the other is degraded (Kawamata and Tomari 2010). This selection seems mostly driven by the thermodynamic stability of both ends forming the duplex (Meijer et al. 2014).

There is emerging interest in performing miRNA profiling in body fluids or tissues in order to identify novel molecular determinants of human diseases (Mitchell et al. 2008; Pulcrano-Nicolas et al. 2018). Such miRNA profiling can

(C) 2019 Thibord et al. This article, published in RNA, is available under a Creative Commons License (Attribution 4.0 International), as described at http://creativecommons.org/licenses/by/4.0/. 
be achieved using hybridization (microarray), next-generation sequencing (NGS), or real time-quantitative polymerase chain reaction (RT-qPCR) techniques. With 2588 known mature miRNAs in humans according to miRBase version 21 (Kozomara and Griffiths-Jones 2014), RT-qPCR would be cumbersome on a genomic scale, but is widely recognized as a gold standard for the validation of few miRNAs. The NGS technology is becoming more popular than microarrays because of its greater detection sensitivity, and higher accuracy in differential expression analysis (Git et al. 2010; Tam et al. 2014). NGS applied to small RNAs revealed a great diversity in the sequences of mature miRNAs originating from the same hairpin. This diversity is mostly attributable to the deletion and addition of nucleotides at the miRNAs' extremities (also known as trimming and tailing events, respectively), due to the activity of terminal nucleotidyl transferases, exoribonucleases, or imprecise cleavage by DROSHA and DICER (Wyman et al. 2011; Neilsen et al. 2012; Ameres and Zamore 2013). To a lesser extent, the ADAR protein acting on double stranded RNAs and responsible for A-to-l editing is also known to target miRNAs (Nishikura 2016). These post-transcriptional editing mechanisms have been shown to affect miRNAs' function and stability (Kawahara et al. 2007; Burroughs et al. 2010; Chiang et al. 2010; Katoh et al. 2015). Lastly, genetic variations have also been shown to contribute to the sequence diversity of miRNAs, and to affect their function and expression (Mencía et al. 2009; Gong et al. 2012; Han and Zheng 2013; Cammaerts et al. 2015). MiRNAs subject to post-transcriptional events and/or genetic variations are generally referred to as isomiRs. In the following, we will use the expression "polymiR" to refer to the subclass of isomiRs harboring genetic polymorphisms in their miRNA sequence.

The first step in the bioinformatics analysis of miRNA sequencing (miRSeq) data consists in aligning sequenced reads to a reference library of mature miRNAs. This step may be challenging because (i) the aforementioned variability of isomiRs could lead to imperfect alignments to the reference library; (ii) sequenced reads may correspond to (fragments of) other molecules (e.g., other small noncoding RNAs like piRNA, tRNA, yRNA...), captured during the preparation of the libraries, that might share a high similarity with miRNAs because of their small length and thus might be confused with miRNAs (Chen and Heard 2013; Heintz-Buschart et al. 2018); (iii) some miRNAs have homologous sequences that are identical or very similar, thus a single read might align ambiguously to multiple reference sequences. In this work, we investigate the impact of the presence of polymorphisms in the sequence of mature miRNAs on their alignment and their expression in the context of miRSeq profiling applied to samples that have also been typed for genome-wide genotype data. This is a situation we anticipate to become rather common with the rise of increasingly affordable genome-wide association stud- ies (GWAS) and the decreasing cost of next-generation exome/genome sequencing techniques. In that context, we developed an original bioinformatics workflow called OPTIMIR, for pOlymorPhism inTegratlon for MIRna data alignment, that integrates genetic information from genotyping arrays or DNA sequencing into the miRSeq data alignment process with the aim of improving the accuracy of polymiRs alignment, while accommodating for other isomiRs detection and ambiguously aligned reads. In addition, OPTIMIR allows to assess the association of genotypes on polymiRs with corresponding polymiRs' expression. OPTIMIR was evaluated in the plasma samples of 391 individuals, part of the MARTHA study (Oudot-Mellakh et al. 2012).

\section{RESULTS}

OPTIMIR is composed of three main steps (Materials and Methods). First, miRSeq data are aligned to a reference library upgraded with sequences integrating alternative alleles of genetic variations. A correction is then applied for ambiguous and unreliable alignments via a scoring approach. Finally, polymiR alignments are evaluated to only retain those that are consistent with input genotypes in case these have been provided by the user. The general workflow was summarized in Figure 1.

\section{MiRNA alignment}

OPTIMIR was evaluated on 391 miRNA sequencing data files totaling 7,390,947,662 sequencing reads. After preprocessing the sequenced reads, that included adapters' removal and selecting only reads with size ranging between 15 and 27 nt, 2,922,446,965 reads (39.54\% of total reads) remained for alignment. 562,040,494 of these reads (19.23\%) were then mapped to mature miRNA reference sequences, of which 10,937,479 (1.95\% of mapped reads) aligned ambiguously to two sequences or more. The application of the OPTIMIR scoring algorithm for alignment disambiguation resulted in a unique solution for $91.6 \%$ of these cross-mapping reads. OPTIMIR computes a score based on the number of editing events that a sequenced read could be compatible with and keeps alignments with the lowest scores (i.e., the lowest number of editing events). For example, if a given read aligns to two reference sequences, perfectly on the first one (score of zero), but with a missing base on the $3^{\prime}$ end (score of one) on the second, then only the first alignment is kept. A score of zero corresponds to a perfect match, and each editing event adds penalties to the score. Modifications in the 5 ' end are more penalizing, as they are less frequently observed. If a cross-mapping read receives an equal score on different alignments, then its weight is divided accordingly to the number of equivalent alignments (see Materials and Methods). For $89.5 \%$ of reads with multiple alignments, 
Input files:

- VCF file containing genetic variants and optional genotype information

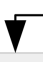

Alignment Library Preparation
Generate new miRNA reference
sequences containing alternative alleles of
variants provided in the VCF file

Alignment

Bowtie2 in local alignment mode

- At least 17 consecutive nucleotides match the reference

- Unmatched extremities are soft-clipped

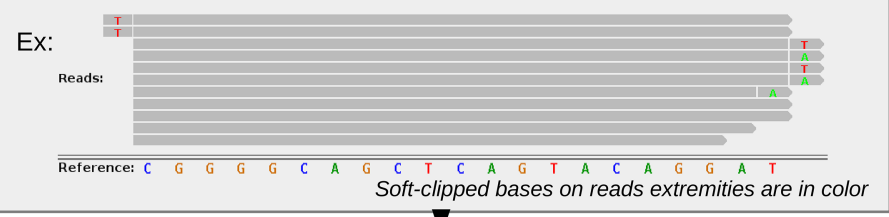

Scoring Algorithm

- For each alignment, compute alignment score $\mathrm{S}_{\mathrm{al}}=\mathrm{N}_{5} \times \mathrm{W}_{5}+\mathrm{N}_{3}{ }^{(a)}$

- Resolution of ambiguous alignments (keep alignment with lowest $\mathrm{S}_{\mathrm{a}}$ )

- Discard alignments with $S_{a l}$ above the specified threshold (set to 9 for this study)

\begin{tabular}{l} 
Genotype Consistency ${ }^{(b)}$ \\
For alignments involving polymiRs: \\
- Check if the read covers the genetic variant \\
- Compare the genotype inferred from the alignment with the genotype \\
provided in VCF \\
- For situation involving a homozygous variant, discard reads aligned to \\
the improper allele \\
- Inconsistency is called if more than $1 \%$ of reads aligned to the polymiR \\
involving an allele not supported by the VCF genotype \\
\hline Output files: \\
- miRNA abundances (3 levels): miRNA level, polymiR level, \\
isomiR level \\
- PolymiRs alignments inconsistent with provided genotypes \\
- Optional : VCF with genotypes inferred from alignment, \\
isomiR distribution, parental hairpins, unresolved \\
ambiguous alignments, mirGFF3
\end{tabular}

FIGURE 1. OPTIMIR workflow. From an optional VCF file and a FASTQ file, OPTIMIR performs the alignment of miRSeq data and results in the generation of abundances files containing the expressions of sequenced miRNAs. (a) The score $\mathrm{S}_{\mathrm{al}}$ is based on the number of editing events observed on the $5^{\prime}$ end and $3^{\prime}$ end of a read ( $N_{5}$ and $N_{3}$, respectively), with a weight $W_{5}$ applied to the $5^{\prime}$ end events (set to four in this study). (b) The genotype consistency is checked only if the user provided genotypes for the variants in the VCF file.

the difference between the two lowest scores was greater than two (Fig. 2A) which would correspond to alignments that differ from each other by at least two modifications in the $3^{\prime}$ end.

After alignment disambiguation, scores ranged from zero to 76 with $98.0 \%$ of alignment scores lower or equal to nine. Beyond this threshold, the quantile distribution curve rapidly increased indicating that scores with higher values are very sparse and suggesting that such alignments with a very high number of editing events are likely improper alignments (Fig. 2B). As a consequence, for the following, we decided to discard any alignment with a score greater than nine. Among the 550,946,055 remain- ing reads, more than $40 \%$ were perfectly aligned or involved templated additions, which were not penalized as these nucleotides are present in the parental hairpin sequence. An additional $40 \%$ of alignments involved reads with a single event in the $3^{\prime}$ end (see Fig. 2C).

\section{IsomiRs distribution}

A total of $197,808,779$ (35.9\%) reads perfectly aligned to mature miRNAs; such reads being generally referred to as canonical miRNAs, and received an alignment score of zero. This confirms previous observations suggesting that a substantial amount of miRNAs are mainly represented by alternative isomiRs (Wallaert et al. 2017; Wu et al. 2018).

The most common observed editing events were on miRNAs' $3^{\prime}$ end, with $\sim 34 \%$ of trimming, $17 \%$ tailing with nontemplated nucleotides, $5 \%$ tailing with templated nucleotides, and a similar proportion of trimming events followed by tailing. The latter modification could also be interpreted as nucleotide variation due to genetic variants, or other less frequent posttranscriptional editing events such as A-to-l editing. It should also be noted that library preparation and sequencing could also contribute to a significant amount of fragment modifications that are falsely detected as isomiRs (Wright et al. 2019).

The $5^{\prime}$ end was much less frequently edited, with $94 \%$ of reads having no editing on this extremity. Nevertheless, the most frequently observed modification on $5^{\prime}$ ends was trimming that affected $4.4 \%$ of all reads. This may be of biological relevance since such trimming could shift the miRNA binding seed that is crucial for the miRNA to bind to its mRNA targets.

The distribution of $3^{\prime}$ and $5^{\prime}$ ends modifications on mapped reads observed over the 391 samples processed by OPTIMIR is shown in Figure 3.

\section{Alignment on polymiRs}

Over all samples, 220,156 reads mapped to 46 polymiRs for a total of 1786 distinct alignments. As detailed in Table 1, 19 polymiRs have reads that aligned to an 


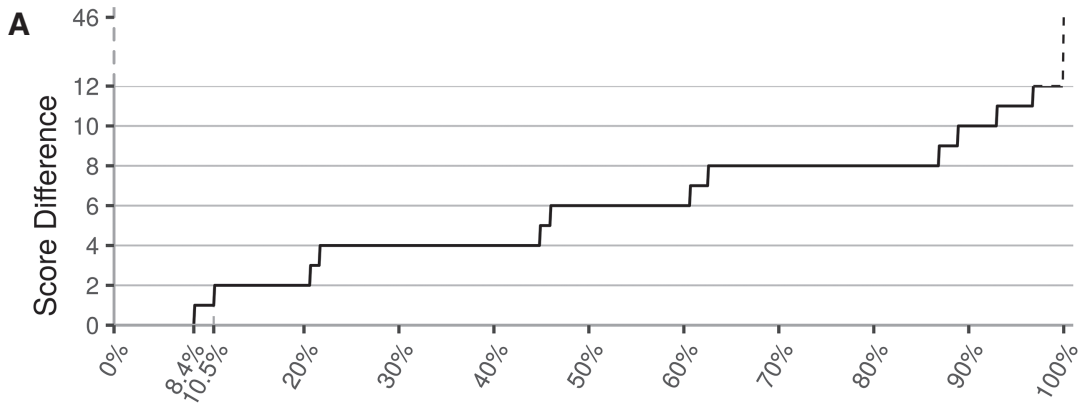

Distribution percentiles : Score difference of ambiguous alignments

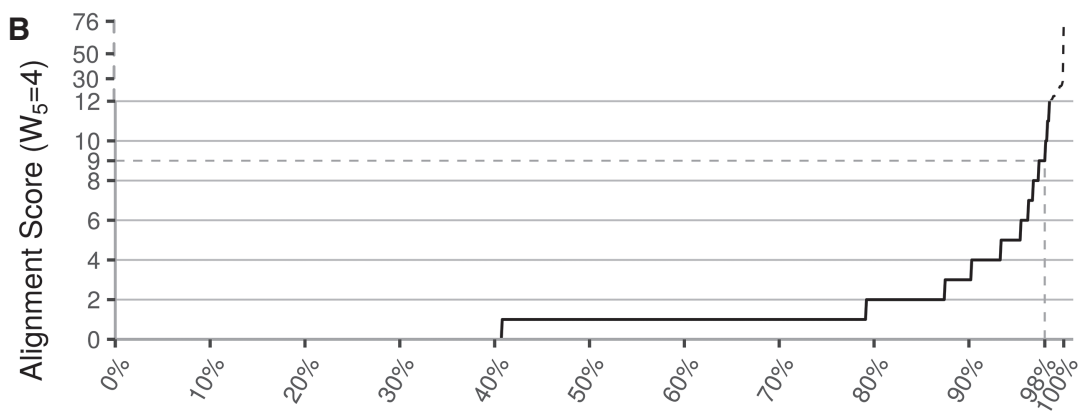

C

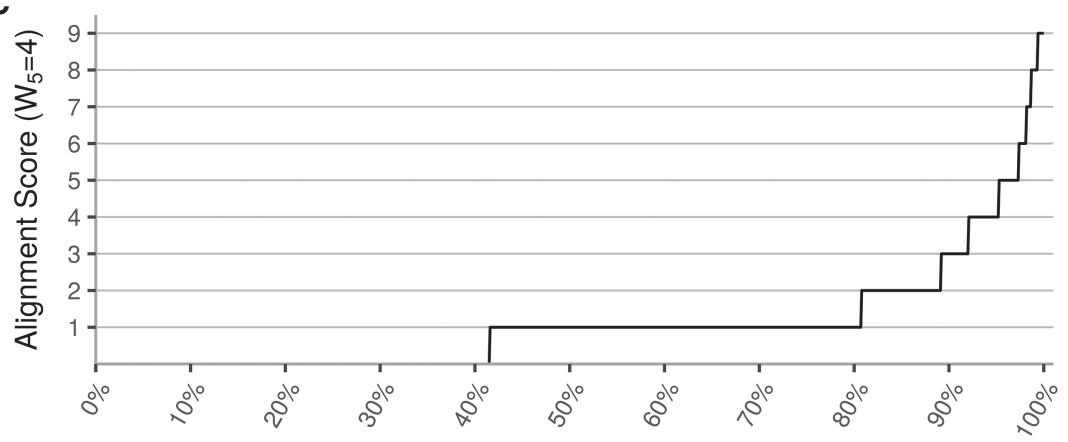

Percentiles of the score distribution

FIGURE 2. Influence of the scoring algorithm. (A) This figure represents the percentile distribution of the score differences between the two best alignments of each cross-mapping read. A total of $8.4 \%$ of cross-mapping reads have a score difference of zero, which means they aligned on different sequences with the same score. These alignments could not be disambiguated. W5 indicates the penalty weight for events in the $5^{\prime}$ end, fixed to four in this study. (B) The alignment score percentile distribution after cross-mapping reads disambiguation represented in percentiles. A total of $98 \%$ of alignments have a score lower or equal to nine. The remaining $2 \%$ of alignments have a score ranging from 10 to 76 , which were categorized as unreliable isomiRs because of the unlikely number of editing events they would have undergone. (C) The same distribution as in $B$ but after removing alignments with a score higher than nine.

alternative sequence that was introduced in the upgraded reference library. Two polymiRs (hsa-miR-6796-3p and hsamiR-1269b) harbor two SNPs, and for both of them only the reference sequence with both common alleles were found to be expressed. Among the remaining 44 polymiRs that harbor only one SNP, 15 were expressed with both alleles. It is important to mention that the allele present in the miRBase reference sequence may not be the most common one (e.g., the rs2155248 and hsa-miR-1304-3p) which may lead to improperly discarding of reads if stringent alignment (with no mismatch allowed) to the original miRBase library is applied.

In total, 155 alignments involving 721 reads $(0.33 \%$ of reads mapped to polymiRs) were found inconsistent with the individual genotypes, among which 145 alignments were supported by less than five reads. These alignments were discarded and not further discussed as they most probably correspond to sporadic mechanisms that mimic genetic variations such as modifications induced during library preparation or sequencing (Wright et al. 2019), or uncommon posttranscriptional editing events (see cMaterials and Methods). The remaining 10 alignments, involving 507 reads spread over 10 samples and five polymiRs, are detailed in Table 2 and further investigated in the next section.

\section{Investigation of inconsistent genotypes}

Situations where a substantial number of reads aligned inconsistently on a polymiR were reported by OPTIMIR to allow for further investigations.

The first case of inconsistent genotype concerns individual PVP28 imputed to be homozygous for the rs12473206-G allele while showing numerous reads mapping to both versions of the hsa-miR-4433b-3p polymiR. Sanger resequencing revealed that this individual is in fact heterozygous for this variant which is much more compatible with the number of observed reads at this locus. A rather similar inconsistent genotype was observed for individual SSP20 but lack of available DNA did not allow us to perform Sanger validation. Lack of DNA also prevented us from investigating deeper the inconsistent genotypes observed for rs72631820/hsa-miR339-3p and rs6771809/hsa-miR-6826-5p.

The inconsistent genotype observed for individual AJA9 at rs6841938 and hsa-miR-1255-5p was challenging. Sanger resequencing confirmed that this individual was indeed homozygous for the rs6841938-G allele although it has numerous reads mapping to both versions of hsa-miR-1255-5p. However, the use of BLAST web-service 

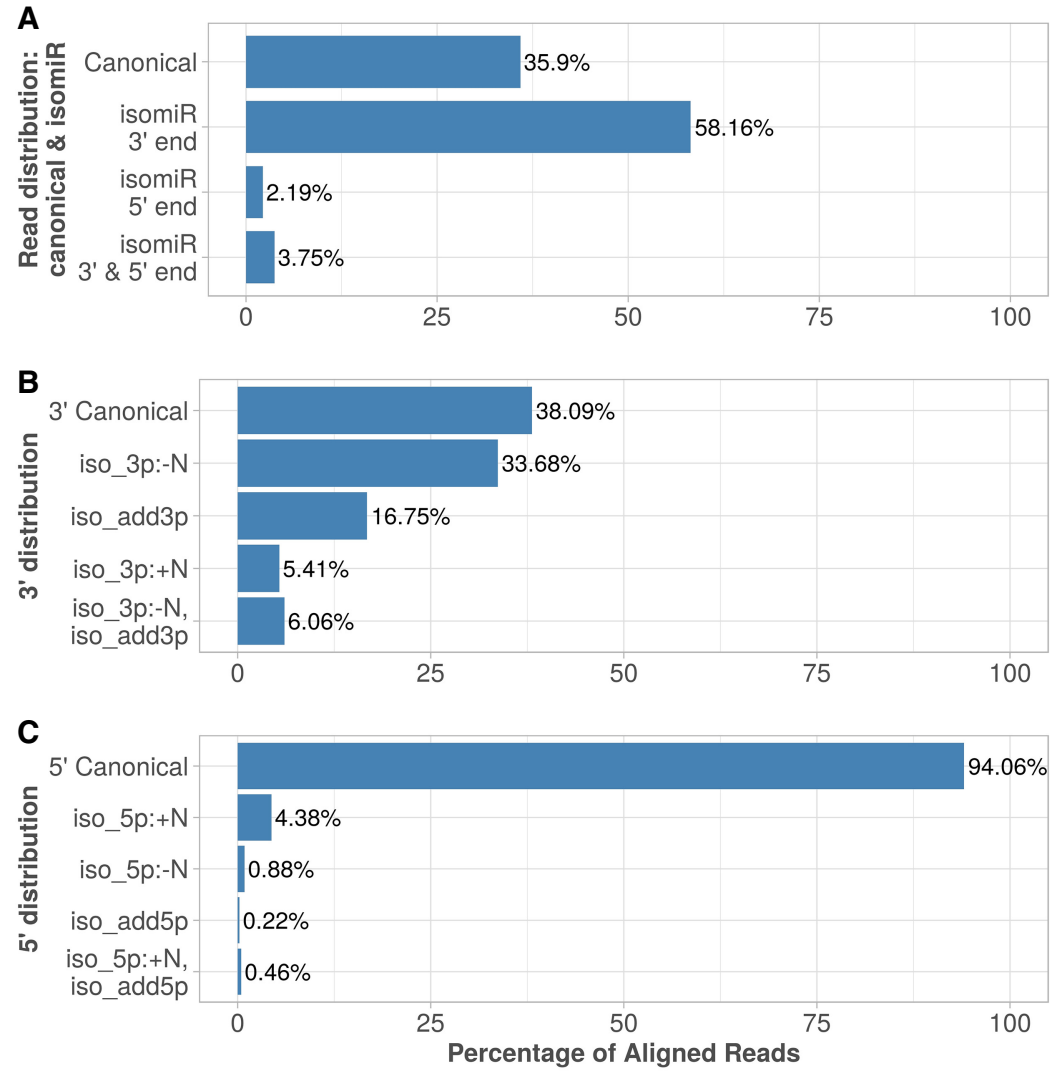

FIGURE 3. IsomiRs profiling. (A) Reads aligned by OPTIMIR distributed between: canonical miRNA (identical to the reference), isomiR $3^{\prime}$ end $/ 5^{\prime}$ end (modifications due to trimming or tailing observed on the $3^{\prime}$ or $5^{\prime}$ end, respectively), and isomiR with both ends edited. (B) Distribution of variations observed on the $3^{\prime}$ end of aligned miRNAs: $3^{\prime}$ Canonical (no modification observed); iso_3p: -N (trimming); iso_3p:+N (templated tailing); iso_add3p (nontemplated tailing); iso_3p: $-\mathrm{N}$, iso_add3p (combination of trimming and nontemplated tailing). (C) Distribution of variations observed on the $5^{\prime}$ end of aligned miRNAs: $5^{\prime}$ Canonical (no modification observed); iso_5p:+N (trimming); iso_5p:-N (templated tailing); iso_add5p (nontemplated tailing); iso_5p:+N,iso_add5p (combination of trimming and nontemplated tailing).

from NCBI (Altschul et al. 1990) revealed that reads aligned on the polymiR sequence containing the rs6841938-A allele perfectly match to the chr1:167,998,699-167, 998,720 region but on the opposite strand where hsamiR-1255-3p should be located. This observation could be compatible with the presence on this opposite strand of an unreported miRNA locus as has already been observed for other miRNAs (e.g., hsa-mir-4433a and hsamir-4433b). Going back to the discarded alignments revealed that 18 other samples homozygous for rs6841938$\mathrm{G}$ had one or two reads that mapped to this opposite strand. These alignments had been discarded since they were supported by less than five reads (see Genotype consistency analysis section in the Materials and Methods).

The last inconsistent genotypes were observed at rs3817551/hsa-miR-7107-3p for five independent individuals. For four of them with available DNA, Sanger resequencing confirmed the initial homozygous genotype for rs3817551-G allele while these individuals were found to express only the hsa-miR-7107-3p version with the $T$ allele. On average, these inconsistent alignments received an alignment score of 1.41 , and none of the reads involved mapped to other sequences. Concerning the samples that had reads aligned on this same reference with a consistent genotype, the average alignment score was very close to 1.47. This score indicates that reads share a high sequence similarity with the hsa-miR-7107-3p, and there is no significant difference between the group with an inconsistent genotype, and the group with a consistent one. BLAST analysis did not enable us to identify any homologous sequence that could explain these observations that still remain to be further investigated in order to be sure that associated reads do originate from hsamiR-7107-3p.

\section{Analysis of polymiRs allele-specific expression}

As shown in Table 1,29 polymiRs with a SNP in their mature sequence were found to be expressed in individuals heterozygotes for this SNP. While one could anticipate that, in heterozygous individuals for such SNP, the polymiRs could have balanced expression (as measured by read counts) of both the reference and alternative sequences, this was hardly observed. Indeed, we observed a strong preference for either the reference or the alternative version of the polymiR in heterozygotes.

Figure 4 shows the alignments of 4 polymiRs involving heterozygous variants according to the vcf genotype. The dotted line represents a theoretical balanced expression between reference and alternative sequences. We can see that, for hsa-miR-1255b-5p/rs6841938 and hsamiR-5189-3p/rs35613341, dots are close to the $y$-axis, which are situations where only the reference allele is expressed. The polymiR with the expression closest to allelic balance for many samples is hsa-miR-4433b-3p but, even then, the average rate of alternative reads across all 147 samples is of 0.8 (see Supplemental Table 4 for details on polymiRs involved in heterozygous situations). As the last case, 65 MARTHA individuals were heterozygous for the rs2925980 variant associated with polymiR hsa-miR7854-3p. These genotypes could be considered as reliable as this SNP was directly typed on the array. Among these 
TABLE 1. Summary information at detected polymiRs

\begin{tabular}{|c|c|c|c|c|c|c|c|c|c|}
\hline \multirow[b]{2}{*}{ polymiR } & \multirow[b]{2}{*}{ rsID } & \multicolumn{3}{|c|}{$\begin{array}{l}\text { Number of samples expressing } \\
\text { the polymiR with genotype }\end{array}$} & \multicolumn{2}{|c|}{$\begin{array}{l}\text { Number of reads } \\
\text { aligned on polymiR } \\
\text { sequence integrating } \\
\text { the allele }\end{array}$} & \multicolumn{2}{|c|}{$\begin{array}{l}\text { Number of } \\
\text { inconsistent reads } \\
\text { removed on the } \\
\text { sequence integrating } \\
\text { the allele }\end{array}$} & \multirow{2}{*}{$\begin{array}{l}\text { Inconsistent } \\
\text { alignments to } \\
\text { investigate }^{\mathrm{b}}\end{array}$} \\
\hline & & $0 / 0$ & $0 / 1$ & $1 / 1$ & Reference & Alternative & Reference & Alternative & \\
\hline hsa-miR-4433b-3p & rs12473206 (C/G) & $223(/ 230)$ & 147 (/147) & $14(/ 14)$ & 63,762 & 96,915 & 4 & 475 & 2 \\
\hline hsa-miR-1255b-5p & rs6841938 (G/A) & $244(/ 323)$ & $48(/ 66)$ & $2(/ 2)$ & 29,642 & 66 & 0 & 52 & 1 \\
\hline hsa-miR-1304-3p & rs2155248 (G/T) & $0(/ 0)$ & $5(/ 7)$ & $256(/ 384)$ & 146 & 11,928 & 4 & 0 & 0 \\
\hline hsa-miR-339-3p & rs72631820 (T/C) & $213(/ 386)$ & $3(/ 5)$ & $0(/ 0)$ & 6293 & 0 & 0 & 21 & 1 \\
\hline hsa-miR-7854-3p & rs2925980 (A/G) & 22 (/159) & $65(/ 167)$ & $35(/ 65)$ & 392 & 2015 & 1 & 4 & 0 \\
\hline hsa-miR-4745-5p & rs10422347 (C/T) & $86(/ 300)$ & $14(/ 81)$ & $0(/ 10)$ & 2330 & 15 & 0 & 0 & 0 \\
\hline hsa-miR-5189-3p & rs35613341 (C/G) & $42(/ 196)$ & $20(/ 161)$ & $0(/ 34)$ & 1315 & 0 & 2 & 1 & 0 \\
\hline hsa-miR-7107-3p & rs3817551 (T/G) & 20 (/157) & 33 (/177) & $0(/ 57)$ & 1104 & 1 & 122 & 0 & 5 \\
\hline hsa-miR-4741 & rs7227168 (C/T) & $40(/ 293)$ & $4(/ 93)$ & $0(/ 5)$ & 695 & 0 & 0 & 0 & 0 \\
\hline hsa-miR-548| & rs13447640 (G/A) & $30(/ 373)$ & $0(/ 18)$ & $0(/ 0)$ & 613 & 0 & 0 & 0 & 0 \\
\hline hsa-miR-3620-5p & rs2070960 (C/T) & $47(/ 348)$ & $4(/ 42)$ & $0(/ 1)$ & 459 & 0 & 0 & 0 & 0 \\
\hline hsa-miR-6826-5p & rs6771809 (T/C) & $11(/ 286)$ & $11(/ 92)$ & $2(/ 13)$ & 199 & 243 & 0 & 21 & 1 \\
\hline hsa-miR-4638-5p & rs146528803 (G/A) & $20(/ 369)$ & $1(/ 22)$ & $0(/ 0)$ & 313 & 0 & 0 & 0 & 0 \\
\hline hsa-miR-3622a-5p & rs66683138 (G/A) & $6(/ 231)$ & 4 (/138) & $0(/ 22)$ & 241 & 24 & 0 & 0 & 0 \\
\hline hsa-miR-4707-3p & rs2273626 (C/A) & $4(/ 86)$ & $6(/ 195)$ & $0(/ 110)$ & 162 & 0 & 0 & 0 & 0 \\
\hline hsa-miR-1269a & rs73239138 (G/A) & 5 (/236) & 2 (/131) & $0(/ 24)$ & 107 & $31.5^{d}$ & 0 & 0 & 0 \\
\hline hsa-miR-4781-3p & rs74085143 (G/A) & 8 (/366) & $0(/ 25)$ & $0(/ 0)$ & 135 & 0 & 0 & 0 & 0 \\
\hline hsa-miR-6763-3p & rs3751304 (C/T) & $1(/ 39)$ & 2 (/153) & 2 (/199) & 86 & 29 & 0 & 0 & 0 \\
\hline hsa-miR-4804-5p & rs266435 (C/G) & $0(/ 7)$ & 3 (/91) & 7 (/293) & 0 & 114 & 1 & 0 & 0 \\
\hline hsa-miR-4999-5p & rs72996752 (A/G) & 5 (/189) & $1(/ 163)$ & $0(/ 39)$ & 86 & 26 & 0 & 1 & 0 \\
\hline hsa-miR-6839-5p & rs7804972 (G/A) & $1(/ 153)$ & $2(/ 196)$ & $0(/ 42)$ & 56 & 0 & 1 & 0 & 0 \\
\hline hsa-miR-4459 & rs73112689 (C/T) & $3(/ 245)$ & 1 (/133) & $0(/ 13)$ & 51 & 0 & 0 & 0 & 0 \\
\hline hsa-miR-585-3p & rs62376935 (C/T) & $0(/ 330)$ & $2(/ 52)$ & 0 (/9) & 2 & 48 & 0 & 0 & 0 \\
\hline hsa-miR-3928-5p & rs5997893 (A/G) & $1(/ 38)$ & $1(/ 157)$ & $1(/ 196)$ & 47 & 9 & 0 & 0 & 0 \\
\hline hsa-miR-548al & rs515924 (A/G) & 0 (/307) & $1(/ 80)$ & $1(/ 4)$ & 0 & 37 & 0 & 0 & 0 \\
\hline hsa-miR-3117-3p & rs12402181 (G/A) & 1 (/288) & $0(/ 97)$ & $0(/ 6)$ & 35 & 0 & 0 & 0 & 0 \\
\hline hsa-miR-3130-3p & rs2241347 (C/T) & $2(/ 254)$ & 3 (/119) & $0(/ 18)$ & 35 & 0 & 0 & 0 & 0 \\
\hline hsa-miR-4532 & rs73177830 (G/A) & $2(/ 344)$ & $0(/ 44)$ & $0(/ 3)$ & 34 & 0 & 0 & 0 & 0 \\
\hline hsa-miR-302c-3p & rs199971565 (del) & $1(/ 371)$ & $0(/ 20)$ & $0(/ 0)$ & 30 & 0 & 0 & 0 & 0 \\
\hline hsa-miR-4772-5p & rs62154973 (C/T) & 3 (/339) & $0(/ 51)$ & $0(/ 1)$ & 25 & 0 & 0 & 0 & 0 \\
\hline hsa-miR-1343-5p & rs2986407 (T/C) & $0(/ 20)$ & $0(/ 111)$ & $2(/ 260)$ & 0 & 24 & 0 & 0 & 0 \\
\hline hsa-miR-548ap-5p & rs4414449 (G/A) & $2(/ 47)$ & $11(/ 180)$ & $1(/ 164)$ & 16 & 4 & 4 & 0 & 0 \\
\hline hsa-miR-6885-5p & rs78293125 (A/G) & $1(/ 341)$ & $0(/ 47)$ & $0(/ 3)$ & 15 & 0 & 0 & 0 & 0 \\
\hline hsa-miR-548h-3p & rs73235381 (C/T) & 3 (/379) & $0(/ 11)$ & $0(/ 1)$ & 14 & 0 & 0 & 0 & 0 \\
\hline hsa-miR-4433a-5p & rs12473206 (C/G) & $1(/ 230)$ & 2 (/147) & $0(/ 14)$ & 12 & 0 & 0 & 0 & 0 \\
\hline hsa-miR-6887-5p & rs1688017 (G/A) & 1 (/152) & 0 (/193) & $0(/ 46)$ & 10 & 0 & 0 & 0 & 0 \\
\hline hsa-miR-4482-5p & rs45596840 (G/A) & 1 (/167) & 0 (/179) & $0(/ 45)$ & 10 & 0 & 0 & 0 & 0 \\
\hline hsa-miR-4520-5p & rs8078913 (C/T) & $0(/ 87)$ & 0 (/198) & $1(/ 106)$ & 0 & 9 & 0 & 0 & 0 \\
\hline hsa-miR-1229-5p & rs2291418 (G/A) & $2(/ 363)$ & $0(/ 27)$ & $0(/ 1)$ & 7 & 0 & 0 & 0 & 0 \\
\hline hsa-miR-6805-3p & rs56312243 (C/T) & 1 (/337) & $0(/ 50)$ & $0(/ 4)$ & 5 & 0 & 0 & 0 & 0 \\
\hline hsa-miR-4302 & rs11048315 (G/A) & 1 (/278) & 2 (/102) & $0(/ 11)$ & 1 & 2 & 0 & 6 & 0 \\
\hline hsa-miR-4520-3p & rs8078913 (C/T) & $0(/ 87)$ & 1 (/198) & $0(/ 106)$ & 1 & 0 & 0 & 0 & 0 \\
\hline hsa-miR-548h-5p & rs9913045 (G/A) & 0 (/143) & 0 (/197) & $0(/ 51)$ & 0 & 0 & 1 & 0 & 0 \\
\hline hsa-miR-6863 & rs12708966 (G/A) & $1(/ 385)$ & $0(/ 6)$ & $0(/ 0)$ & 1 & 0 & 0 & 0 & 0 \\
\hline hsa-miR-6796-3p & $\begin{array}{l}\text { rs3745199 (C/G) } \\
\text { rs3745198 (C/G) }\end{array}$ & $\begin{array}{l}3(/ 131) \\
3(/ 131)\end{array}$ & $\begin{array}{l}0 \text { (/193) } \\
0 \text { (/190) }\end{array}$ & $\begin{array}{l}0(/ 67) \\
0(/ 70)\end{array}$ & 56 & $0^{c}$ & 0 & 0 & 0 \\
\hline
\end{tabular}


TABLE 1. Continued

\begin{tabular}{|c|c|c|c|c|c|c|c|c|c|}
\hline \multirow[b]{2}{*}{ polymiR } & \multirow[b]{2}{*}{ rsID } & \multicolumn{3}{|c|}{$\begin{array}{l}\text { Number of samples expressing } \\
\text { the polymiR with genotype }\end{array}$} & \multicolumn{2}{|c|}{$\begin{array}{l}\text { Number of reads } \\
\text { aligned on polymiR } \\
\text { sequence integrating } \\
\text { the allele }\end{array}$} & \multicolumn{2}{|c|}{$\begin{array}{l}\text { Number of } \\
\text { inconsistent reads } \\
\text { removed on the } \\
\text { sequence integrating } \\
\text { the allele }\end{array}$} & \multirow{2}{*}{$\begin{array}{l}\text { Inconsistent } \\
\text { alignments to } \\
\text { investigate }^{b}\end{array}$} \\
\hline & & $0 / 0$ & $0 / 1$ & $1 / 1$ & Reference & Alternative & Reference & Alternative & \\
\hline \multirow[t]{2}{*}{ hsa-miR-1269b } & $\begin{array}{l}\text { rs12451747 (A/C) } \\
\text { rs7210937 (G/C) }\end{array}$ & $\begin{array}{r}0(/ 82) \\
1(/ 326)\end{array}$ & $\begin{array}{r}1(/ 171) \\
0(/ 60)\end{array}$ & $\begin{array}{r}0(/ 138) \\
0(/ 5)\end{array}$ & $31.5(d)$ & $0^{c}$ & 0 & 0 & 0 \\
\hline & TOTAL & 1062 & 400 & 324 & 108574.5 & 111540.5 & 140 & 581 & 10 \\
\hline \multicolumn{10}{|c|}{$\begin{array}{l}\text { aBetween parentheses is the total number of samples carrying the variant with homozygous reference allele }(0 / 0) \text {, heterozygous }(0 / 1) \text {, or homozygous alter- } \\
\text { native allele (1/1). } \\
\text { b Alignments with a high number of inconsistent reads (higher than } 1 \% \text { of the sum of reads gathered by the polymiR sequences), which is incompatible with } \\
\text { sporadic events replicating a genetic variant. } \\
{ }^{c} \text { For multivariant polymiRs, reads did not map to any of the sequence integrating alternative haplotypes. } \\
{ }^{d} \text { The sequence of hsa-miR- } 1269 \text { a integrating the rs } 73239138-A \text { allele is identical to the hsa-miR-1269b sequence. Hence, each of the } 63 \text { reads that cross- } \\
\text { mapped on both sequences received a count of } 1 / 2 \text { on each sequence. }\end{array}$} \\
\hline
\end{tabular}

65 individuals, 54 expressed only the alternative version of polymiR hsa-miR-7854-3p, and the remaining 11 expressed both polymiR versions with a mean ratio of 0.96 in favor of the alternative allele.

Finally, we used the RNAfold program (Lorenz et al. 2011) to predict the secondary structure of the 29 primiRNA (i.e., hairpins) induced by the presence of a SNP in the polymiR sequence (Supplemental Fig. S1). Most genetic variations create either a new bulge, or a wobble pairing, or have no impact on the secondary structure. A notable exception relates to rs35613341 located on the hsa-miR-5189-3p where the $G$ allele completely changed the secondary structure of the hairpin (see Supplemental Fig. S1[u]) making it difficult to access for the DICER and DROSHA machinery. In MARTHA samples, 161 individuals were heterozygous and 34 homozygous for the rs35613341-G allele. None of these individuals were found to express the alternative sequence of polymiR hsa-miR5189-3p which could support the hypothesis that the rs35613341-G allele impacts the maturation of this miRNA.

Lastly, by the completion of the OPTIMIR pipeline (with a scoring threshold of nine as mentioned above and keeping only genotype consistent reads) on MARTHA samples, $7.45 \%$ of sequenced reads were aligned. This value had to be compared with $7.68 \%$ and $8.24 \%$ obtained by two other recent pipelines for miRSeq data, sRNAnalyzer (Wu et al. 2017) and miRge (Baras et al. 2015), respectively, executed using default parameters. These discrepancies could be explained by the different alignment strategies implemented by these tools, which by default allow up to two mismatches for read alignment, while OPTIMIR does not allow any mismatch in aligning sequenced reads and relies on local alignment to capture isomiRs with modified extremities (see OPTIMIR description). When we ran

TABLE 2. Inconsistent genotypes unlikely due to sequencing errors

\begin{tabular}{|c|c|c|c|c|c|c|c|c|c|}
\hline polymiR & rsID & $\begin{array}{l}\text { Alleles: } \\
\text { reference, } \\
\text { alternative }\end{array}$ & MAF & RSO & SAMPLE & $\begin{array}{l}\text { Genotype } \\
\text { from vcf }\end{array}$ & $\begin{array}{l}\text { Counts } \\
\text { reference }\end{array}$ & $\begin{array}{l}\text { Counts } \\
\text { alternative }\end{array}$ & $\begin{array}{c}\text { SANGER } \\
\text { validation }\end{array}$ \\
\hline hsa-miR-4433b-3p & rs12473206 & $C / G$ & 0.23 & 0.99 & PVP28 & $\mathrm{C} / \mathrm{C}$ & 84 & 333 & $\mathrm{G} / \mathrm{C}$ \\
\hline hsa-miR-4433b-3p & rs12473206 & $\mathrm{C} / \mathrm{G}$ & 0.23 & 0.99 & SSP2O & $\mathrm{C} / \mathrm{C}$ & 135 & 8 & N.A. \\
\hline hsa-miR-339-3p & rs72631820 & $\mathrm{T} / \mathrm{C}$ & 0.009 & 0.98 & CED24 & $\mathrm{T} / \mathrm{T}$ & 139 & 7 & N.A. \\
\hline hsa-miR-6826-5p & rs6771809 & $\mathrm{T} / \mathrm{C}$ & 0.14 & 0.98 & DTD19 & $\mathrm{T} / \mathrm{T}$ & 1 & 20 & N.A. \\
\hline hsa-miR-1255b-5p & rs6841938 & $\mathrm{G} / \mathrm{A}$ & 0.09 & 0.96 & AJA9 & $\mathrm{G} / \mathrm{G}$ & 92 & 28 & $\mathrm{G} / \mathrm{G}$ \\
\hline hsa-miR-7107-3p & rs3817551 & $\mathrm{T} / \mathrm{G}$ & 0.36 & 0.96 & MSM20 & $\mathrm{G} / \mathrm{G}$ & 27 & 0 & N.A. \\
\hline hsa-miR-7107-3p & rs3817551 & $\mathrm{T} / \mathrm{G}$ & 0.36 & 0.96 & MCC20 & $\mathrm{G} / \mathrm{G}$ & 15 & 0 & $\mathrm{G} / \mathrm{G}$ \\
\hline hsa-miR-7107-3p & rs3817551 & $\mathrm{T} / \mathrm{G}$ & 0.36 & 0.96 & NFN22 & $\mathrm{G} / \mathrm{G}$ & 23 & 0 & $\mathrm{G} / \mathrm{G}$ \\
\hline hsa-miR-7107-3p & rs3817551 & $\mathrm{T} / \mathrm{G}$ & 0.36 & 0.96 & NHN19 & $\mathrm{G} / \mathrm{G}$ & 25 & 0 & $\mathrm{G} / \mathrm{G}$ \\
\hline hsa-miR-7107-3p & rs3817551 & $\mathrm{T} / \mathrm{G}$ & 0.36 & 0.96 & GLG20 & $\mathrm{G} / \mathrm{G}$ & 17 & 0 & $\mathrm{G} / \mathrm{G}$ \\
\hline
\end{tabular}

$M A F$, minor allele frequency. RSQ, imputation quality criteria ( $r 2)$. 


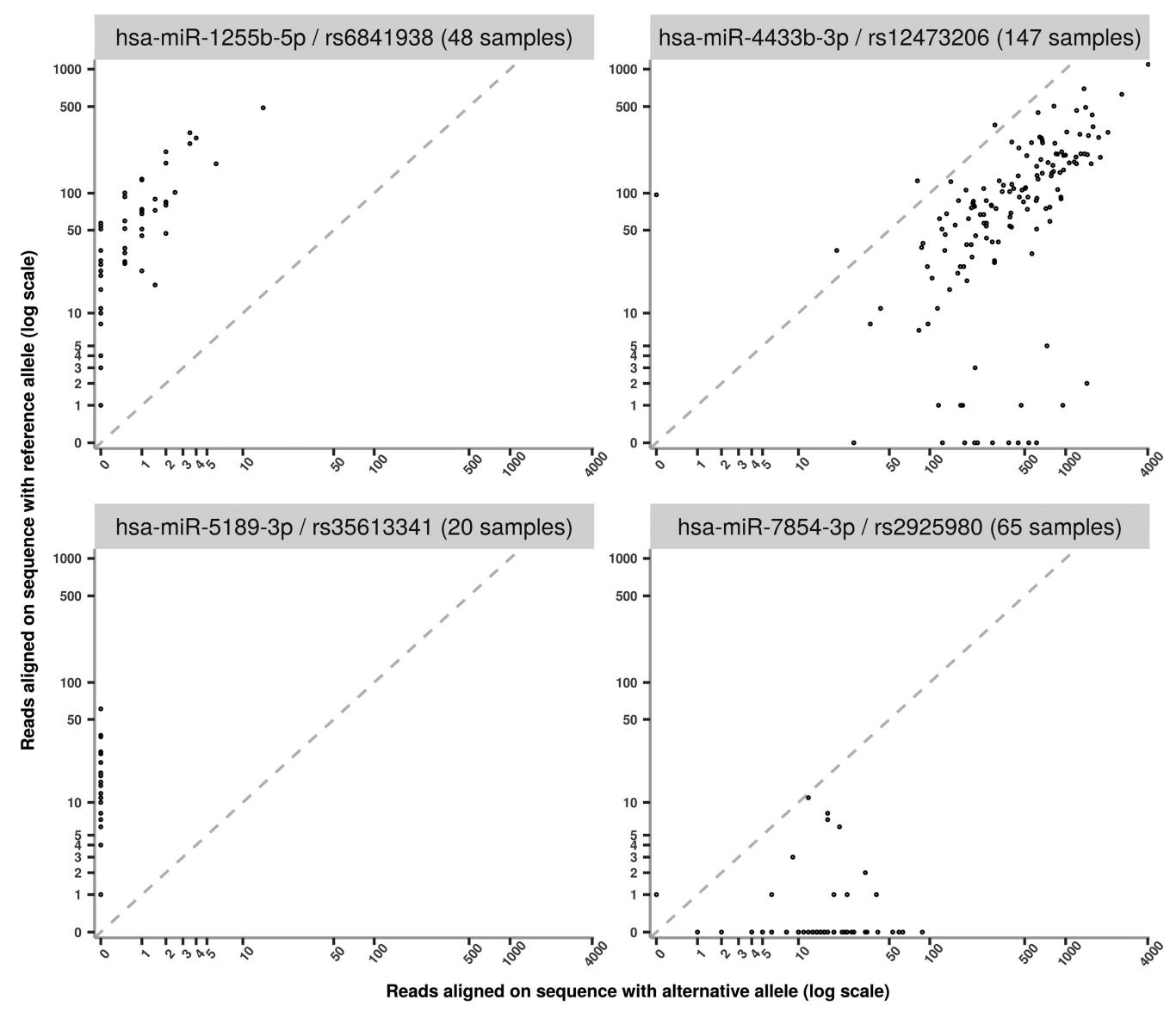

FIGURE 4. Examples of allele imbalanced expressions observed at four polymiRs. On the $x$-axis is shown the expression of the alternative allele, while the $y$-axis represents the expression of the reference allele. Expressions were $\log _{10}$-transformed.

sRNAnalyzer with only one mismatch allowed, the percentage of aligned reads dropped to $7.38 \%$ (compared to $7.68 \%$ with two mismatches). Of note, it was not possible to modify the number of authorized mismatches in miRge.

The files generated by OPTIMIR include (i) global abundances of miRNAs (counts of isomiRs and polymiRs are merged with the reference mature sequences' counts); (ii) specific abundances for each polymiR sequence; (iii) specific abundances for each isomiR sequence; (iv) alignments that are inconsistent with provided genotypes; ( $v$ ) two annotation files containing details on templated nucleotides and alignments that could not be disambiguated. In addition, OPTIMIR allows to generate its results into the recently developed mirGFF3 format (Desvignes et al. 2018) aimed at unifying results of any miRSeq data analysis.

\section{DISCUSSION}

In this work, we propose a novel algorithm, OPTIMIR, for aligning miRNA sequences obtained from NGS and we applied it to plasma samples of 391 individuals from the MARTHA study. Borrowing some ideas from other align- ment pipelines such as the addition of new sequences to the reference library corresponding to allelic versions of polymiRs (Baras et al. 2015; Russell et al. 2018) and a scoring strategy for handling cross-mapping reads (Urgese et al. 2016) or for discarding unlikely isomiRs (Bofill-De Ros et al. 2018), OPTIMIR has two features that make it unique. First, OPTIMIR is based on a scoring strategy that incorporates biological knowledge on miRNA editing to identify the most likely alignment in the presence of cross-mapping reads. Second, OPTIMIR allows the user to provide genotype information, in particular data obtained from genome-wide genotyping arrays, to improve alignment accuracy. This option revealed several interesting observations when OPTIMIR was applied to MARTHA plasma samples.

First, it allowed the identification of improperly imputed genotypes despite overall good imputation quality. Second, it suggested the existence of a new miRNA not indexed in the miRBase v21 database that would be located on the opposite strand of the hsa-miR-1255b-5p. Thirdly, it suggested that reads aligned to hsa-miR-7107-3p are likely false alignments and would more likely come from other noncoding fragments that share sequence similarity with 
hsa-miR-7107-3p. These last two hypotheses would need to be further validated but this is out of the scope of the bioinformatics workflow described in the current work. Even more interesting was the study of polymiRs' expressions in heterozygous individuals for SNPs on these polymiRs. OPTIMIR clearly showed that plasma allelic expression of polymiRs is unbalanced for most polymiRs. One allelic version of a polymiR is much more expressed than the other and this is not necessarily the one carrying the most common allele. This observation is consistent with previous works showing that SNPs in (pri-) miRNA sequences can influence miRNA expression through their impact on the DROSHA/DICER RNases' machinery (Duan et al. 2007; Cammaerts et al. 2015). Epigenetic mechanisms could also explain the allelic imbalance expression of miRNAs (Morales et al. 2017).

Several limitations shall however be acknowledged. OPTIMIR requires to fix two parameters, a weight $W_{5}$ for penalizing $5^{\prime}$ end editing event (set to four in the current application) and a score threshold (set to nine here) to discard unreliable alignments. The former tends to have little impact on the general findings (see Supplemental Table S2), while the second may be study-specific and may depend on the study sample-size and the kind of tissue analyzed. These parameters can be easily modified by the user. There is so far no gold standard program for miRNA alignment analysis but our preliminary study suggests that OPTIMIR aligns slightly less reads than two other recently proposed software, sRNAnalyzer (Wu et al. 2017) and miRge (Baras et al. 2015). This is likely due to the higher number of mismatches allowed by the latter two for aligning reads while OPTIMIR tends to be more stringent. Without extensive investigations including experimental validations, it is not possible to really appreciate which alignments are the correct ones.

Finally, several improvements could be considered such as (i) the integration of A-to-l editing events in the definition of our reference library and of our scoring strategy, even if we anticipate that it might be difficult to distinguish these rare events from sequencing errors and (ii) the extension of the OPTIMIR workflow to analyze other small coding RNAs (e.g., piRNA and tRNA, rRNA, snRNA, or yRNA derived fragments) that are generally sequenced together with miRNAs in a miRseq profiling. Applications to other tissues from the samples processed for miRSeq data deserve to be conducted to generalize the findings observed in the current plasma samples.

\section{MATERIALS AND METHODS}

\section{The MARTHA data set}

The MARseille Thrombosis Assocation study is a collection of patients with venous thrombosis (VT) recruited at the La Timone Hospital (Marseille, France) between 1994 and 2005 and aimed at identifying novel molecular determinants for VT and its associated endophenotypes (Oudot-Mellakh et al. 2012; Dick et al. 2014).

For the present study, 391 MARTHA participants with available plasma samples were processed for plasma miRNA profiling through miRSeq. These individuals had been previously typed for genome-wide genotyping arrays and imputed for single nucleotide polymorphisms (SNPs) available in the $1000 \mathrm{G}$ reference database (Germain et al. 2015).

MiRNA extraction and preparation followed the same protocol as the one previously described in Roux et al. (2018). Briefly, from $400 \mu \mathrm{L}$ of plasma, total RNA was first extracted using the miRNeasy serum/plasma kit for Qiagen. MiRNA libraries were then prepared using the NEBNext Multiplex Small RNA Library Preparation Set for adapter ligation and $P C R$, with adapter sequences GATCGGAAGAGCACACGTCTGAACTCCAGTCAC ( 3 ' adapter) and CGACAGGTTCAGAGTTCTACAGTCCGACGATC ( 5 ' adapter) followed by a size selection using AMPure XP beads. Pools of equal quantity of 24 purified libraries were constructed and tagged with different indexes. Pools were then sequenced using a $75 \mathrm{bp}$ single-end strategy on an Illumina NextSeq500 instrument.

\section{The OPTIMIR workflow}

\section{Alignment}

Prealignment data processing. $3^{\prime}$ adapters were removed using cutadapt (Martin 2011) with a base quality filter set to 28 . Remaining reads with sequence length between 15 and 27, which generally correspond to miRNA sequences, were then kept for alignment. Note that identical reads were collapsed together to decrease the computational burden associated with processing $n$ times $n$ identical reads.

Definition of an alignment reference library. Read alignment generally starts by the selection of a reference library to which reads shall be aligned. The miRBase 21 database (Kozomara and Griffiths-Jones 2014) containing known human mature miRNAs is usually adopted for miRSeq data. We first upgrade this reference library by adding new sequences corresponding to the alternate forms of polymiRs showing genetic polymorphisms in their mature sequence as previously proposed (Baras et al. 2015; Russell et al. 2018). In case a polymiR contains more than one polymorphism, new sequences corresponding to all possible haplotypes are generated. These variants, that are provided by the OPTIMIR's user in a vcf format file (Danecek et al. 2011), are mapped to miRNAs using miRBase miRNA coordinates file (i.e., positions of miRNAs on the human reference genome). The generation of new sequences is automated via a standalone python script provided with the OPTIMIR pipeline.

For the current application to MARTHA GWAS data, we identified 88 single nucleotide polymorphisms (SNPs) for which we have a reliable genotype data, defined as SNPs with imputation $r^{2}>0.8$. Note that some SNPs may map to two distinct miRNAs if the latter are transcribed from opposite strands. Some miRNAs may also have more than one SNP in their sequence. In our application, five SNPs mapped to two distinct miRNAs and three miRNAs contained more than one SNP (see Supplemental 
Figure $\mathrm{S} 2$ for examples). As a result, the reference library was upgraded with 96 new alternative sequences corresponding to all possible haplotypes derived from the 90 identified polymiRs.

Read alignment process. For read alignment, we opted for the bowtie2 software (Langmead and Salzberg 2012) that can handle trimming and tailing events at the reads' extremities via its local alignment mode which has been shown to be efficient for miRSeq data alignment (Ziemann et al. 2016). Only reads with a sequence of at least 17 consecutive nucleotides (defined as the alignment seed) that perfectly match with the reference library are kept in the analysis (see Supplemental Table S1 for details concerning the choice of the seed value and its consequences on isomiR detection). In summary, the OPTIMIR pipeline does not authorize any mismatch in the central sequence of a read but allows variations at its extremities to address post-transcriptional editing. The handling of miRNAs with genetic variations is addressed by the use of the upgraded reference library described above. For miRNAs that underwent tailing events, or trimming events followed by tailing events, additional bases exceeding or differing from the reference are soft-clipped and do not participate in the alignment. With a limited read length of $27 \mathrm{nt}$, the maximum number of bases that can be soft-clipped was set to 10 . Reads were allowed to align to multiple reference sequences in order to take into account the different mature miRNAs with similar sequences from which they could originate. Finally, we did not allow reverse complement alignment as small RNAs were first ligated with different $5^{\prime}$ and $3^{\prime}$ adapters before single-end sequencing, which implies that RNA strands were sequenced in only one direction.

\section{Resolution of ambiguous alignments}

To handle multiple ambiguous alignments, OPTIMIR integrated a scoring algorithm aimed at identifying the most plausible alignment(s) while discarding likely erroneous ones. Of note, beforehand, for reads mapping to a mature miRNA that can be produced by two different pri-miRNAs (e.g., hsa-miR-1255b-5p can originate from hsa-mir-1255b-1 or hsa-mir-1255b-2 located on chromosome 4 and 1, respectively), we used the information on templated tailed nucleotides (i.e., nucleotides in the primiRNA sequences (also available in the miRBase 21) that surround the mature miRNA sequence) to deduce from which locus these reads might come from. This information, that has no impact on the alignment per se, is stored in an output file (named expressed_hairpins.annot).

Each alignment was assigned a score based on the number of trimming and tailing events that could make a given read perfectly match with a mature miRNA sequence. Since trimming and tailing are frequently observed in the $3^{\prime}$ end of miRNAs but rare in the $5^{\prime}$ end (Neilsen et al. 2012; Wu et al. 2018), a more penalizing weight was applied on events observed on the $5^{\prime}$ end. Alignments with a lower score would be considered as more reliable as they would correspond to a miRNA with less editing events. The alignment score is calculated as follows:

$$
\text { Alignment score }=N_{5} \times W_{5}+N_{3},
$$

where $N_{5}$ and $N_{3}$ represent the number of editing events observed on the $5^{\prime}$ and $3^{\prime}$ extremities of the read, respectively. $W_{5}$ is the weight applied to the events observed on the $5^{\prime}$ end. Several
$W_{5}$ values were tested and their impact on the alignment results are shown in Supplemental Table S2. Finally, for our application, $W_{5}$ was set to four in order to resolve as many ambiguous alignments as possible without penalizing too many $5^{\prime}$ events compared to $3^{\prime}$ events as they represent $\sim 60 \%$ of aligned reads. Templated tailed nucleotides do not count as editing events as they tend to validate the parentage of a read to its reference. These templated nucleotides are most likely the result of imprecise cleavage by DROSHA and DICER. However, they might occasionally result from the action of the terminal nucleotidyl transferase that adds the same nucleotides as those surrounding the original sequence, in which case they cannot be distinguished.

By the end of the scoring algorithm, the alignment with the lowest score was retained. In case of an ambiguous read with $n$ possible alignments having the same score, all alignments are kept and assigned to a weight of $1 / n$, and corresponding alignments are listed in an output file (named remaining_ambiguous.annot).

Of note, bowtie2 also integrates an alignment score. However, this scoring is general and does not integrate the biological knowledge on editing events specific to miRNAs. The OPTIMIR scoring algorithm also differs from the one recently proposed in IsomiR-SEA pipeline (Urgese et al. 2016) which is based on the number of observed mismatches and the difference in size between a given read and the reference mature miRNAs.

\section{Genotype consistency analysis}

In case users provide genetic information for individuals that have been miRSeq profiled, the last step of the OPTIMIR workflow is to provide a comparison analysis of the genotype data provided by the user (in a standard vcfformat) and the genotype data that could be inferred from the sequenced reads aligned to polymiRs. For an individual whose reads aligned onto a polymiR sequence that harbors the alternate allele of a SNP, consistency will be called if this individual is either heterozygous or homozygous for this allele in the provided vcf genotype file. Inconsistent alignments are discarded but saved in an output file (named inconsistents.sam).

Indeed, it may occur that some reads align to a polymiR sequence that harbors a given allele in an individual that is not expected to carry it. This could be due to modifications induced during library preparation or sequencing that mimic genetic variations (Wright et al. 2019). However, for a given individual and a given polymiR, if this event is observed for a large number of reads, another explanation must be looked for. For instance, this could occur when reads originate from sequenced fragments of other small noncoding RNAs that share a high similarity with a polymiR. Such situations are detailed in a separate output (named consistency_table.annot).

To detect these situations, we set up a threshold based on the number of reads aligned to each allele of the polymiR: if the presence of an alternative allele is supported by more than $1 \%$ of the total reads (i.e., reads with the reference or alternative allele) that aligned to its associated polymiR, then this allele is considered as plausible. For example, given a polymiR with 980 reads aligned to the sequence integrating the reference allele and 20 reads aligned to the sequence integrating the alternative allele, then the percentage of reads supporting the presence of the alternative allele is $2 \%$. Such a situation would then be considered inconsistent for an individual genotyped as homozygous for the reference allele and would deserve to be further investigated. 
Note that, when polymiRs have less than 500 aligned reads in total, a threshold of five supporting reads instead of a percentage of $1 \%$ was used. These parameters can be modified by the users.

Care is needed to call inconsistency when a polymiR may have homologous mature sequences and one of them is polymorphic. For example, the mature miRNA hsa-miR-1255b-5p can originate either from the pri-miRNA hsa-miR-1255b-1 located on chromosome 4 or from hsa-mir-1255b-2 located on chromosome 1. However, only the chromosome 4 copy contains a variant. If reads with the alternate allele can easily be deduced to originate from hsa-miR-1255b-1, reads with the reference allele can come from both chromosome 1 and 4 copies. As a consequence, homozygous carriers of the alternate allele can still have reads mapping to chromosome 1 copies and such reads shall not be considered as inconsistent. We have listed in Supplemental Table S3 all mature miRNAs that have multiple pri-miRNAs sequences and tagged those that are polymorphic.

\section{SUPPLEMENTAL MATERIAL}

Supplemental material is available for this article.

\section{ACKNOWLEDGMENTS}

F.T. and M.R. were financially supported by the GENMED Laboratory of Excellence on Medical Genomics, Agence Nationale de la Recherche (ANR-10-LABX-0013). D-A.T. was financially supported by the "EPIDEMIOM-VTE" Senior Chair from the Initiative of Excellence of the University of Bordeaux. MiRNA sequencing in the MARTHA study was performed on the iGenSeq platform (ICM Institute, Paris) and supported by a grant from the European Society of Cardiology for Medical Research Innovation.

Received November 26, 2018; accepted February 21, 2019.

\section{REFERENCES}

Altschul SF, Gish W, Miller W, Myers EW, Lipman DJ. 1990. Basic local alignment search tool. J Mol Biol 215: 403-410. doi:10.1016/ S0022-2836(05)80360-2

Ameres SL, Zamore PD. 2013. Diversifying microRNA sequence and function. Nat Rev Mol Cell Biol 14: 475-488. doi:10.1038/ nrm3611

Baras AS, Mitchell CJ, Myers JR, Gupta S, Weng L-C, Ashton JM, Cornish TC, Pandey A, Halushka MK. 2015. miRge-a multiplexed method of processing small RNA-seq data to determine microRNA entropy. PLoS One 10: e0143066. doi:10.1371/journal.pone .0143066

Bartel DP. 2004. MicroRNAs: genomics, biogenesis, mechanism, and function. Cell 116: 281-297. doi:10.1016/S0092-8674(04)00045-5

Bofill-De Ros X, Chen K, Chen S, Tesic N, Dusan R, Skundric N, Nessic S, Varjacic V, Williams EH, Malhotra R, et al. 2018. QuagmiR: a cloud-based application for isomiR big data analytics. Bioinformatics doi:10.1093/bioinformatics/bty843

Burroughs AM, Ando Y, de Hoon MJL, Tomaru Y, Nishibu T, Ukekawa R, Funakoshi T, Kurokawa T, Suzuki H, Hayashizaki Y, et al. 2010. A comprehensive survey of $3^{\prime}$ animal miRNA modification events and a possible role for $3^{\prime}$ adenylation in modulating miRNA targeting effectiveness. Genome Res 20: 1398-1410. doi:10.1101/gr.106054.110
Cammaerts S, Strazisar M, De Rijk P, Del Favero J. 2015. Genetic variants in microRNA genes: impact on microRNA expression, function, and disease. Front Genet 6: 186. doi:10.3389/fgene.2015 .00186

Chen CJ, Heard E. 2013. Small RNAs derived from structural non-coding RNAs. Methods 63: 76-84. doi:10.1016/j.ymeth.2013.05.001

Chiang HR, Schoenfeld LW, Ruby JG, Auyeung VC, Spies N, Baek D, Johnston WK, Russ C, Luo S, Babiarz JE, et al. 2010. Mammalian microRNAs: experimental evaluation of novel and previously annotated genes. Genes Dev 24: 992-1009. doi:10.1101/gad .1884710

Danecek P, Auton A, Abecasis G, Albers CA, Banks E, DePristo MA, Handsaker RE, Lunter G, Marth GT, Sherry ST, et al. 2011. The variant call format and VCFtools. Bioinformatics 27: 2156-2158. doi:10.1093/bioinformatics/btr330

Desvignes T, Loher P, Eilbeck K, Ma J, Urgese G, Fromm B, Sydes J, Aparicio-Puerta E, Barrera V, Espin R, et al. 2018. Unification of miRNA and isomiR research: the mirGFF3 format and the mirtop API. bioRxiv doi:10.1101/505222

Dick KJ, Nelson CP, Tsaprouni L, Sandling JK, Aïssi D, Wahl S, Meduri E, Morange P-E, Gagnon F, Grallert H, et al. 2014. DNA methylation and body-mass index: a genome-wide analysis. Lancet 383: 1990-1998. doi:10.1016/S0140-6736(13)62674-4

Duan R, Pak C, Jin P. 2007. Single nucleotide polymorphism associated with mature miR-125a alters the processing of pri-miRNA. Hum Mol Genet 16: 1124-1131. doi:10.1093/hmg/ddm062

Filipowicz W, Bhattacharyya SN, Sonenberg N. 2008. Mechanisms of post-transcriptional regulation by microRNAs: are the answers in sight? Nat Rev Genet 9: 102-114. doi:10.1038/nrg2290

Germain M, Chasman DI, de Haan H, Tang W, Lindström S, Weng L-C, de Andrade M, de Visser MCH, Wiggins KL, Suchon P, et al. 2015. Meta-analysis of 65,734 individuals identifies TSPAN15 and SLC44A2 as two susceptibility loci for venous thromboembolism. Am J Hum Genet 96: 532-542. doi:10.1016/j.ajhg.2015.01.019

Git A, Dvinge H, Salmon-Divon M, Osborne M, Kutter C, Hadfield J, Bertone P, Caldas C. 2010. Systematic comparison of microarray profiling, real-time PCR, and next-generation sequencing technologies for measuring differential microRNA expression. RNA 16: 991-1006. doi:10.1261/rna.1947110

Gong J, Tong Y, Zhang H-M, Wang K, Hu T, Shan G, Sun J, Guo A-Y. 2012. Genome-wide identification of SNPs in microRNA genes and the SNP effects on microRNA target binding and biogenesis. Hum Mutat 33: 254-263. doi:10.1002/humu.21641

Han M, Zheng Y. 2013. Comprehensive analysis of single nucleotide polymorphisms in human microRNAs. PLoS One 8: e78028. doi:10.1371/journal.pone.0078028

Heintz-Buschart A, Yusuf D, Kaysen A, Etheridge A, Fritz JV, May P, de Beaufort C, Upadhyaya BB, Ghosal A, Galas DJ, et al. 2018. Small RNA profiling of low biomass samples: identification and removal of contaminants. BMC Biol 16: 52. doi:10.1186/s12915-0180522-7

Katoh T, Hojo H, Suzuki T. 2015. Destabilization of microRNAs in human cells by $3^{\prime}$ deadenylation mediated by PARN and CUGBP1. Nucleic Acids Res 43: 7521-7534. doi:10.1093/nar/gkv669

Kawahara Y, Zinshteyn B, Sethupathy P, lizasa H, Hatzigeorgiou AG, Nishikura K. 2007. Redirection of silencing targets by adenosineto-inosine editing of miRNAs. Science 315: 1137-1140. doi:10 .1126 /science. 1138050

Kawamata T, Tomari Y. 2010. Making RISC. Trends Biochem Sci 35: 368-376. doi:10.1016/j.tibs.2010.03.009

Kim VN, Han J, Siomi MC. 2009. Biogenesis of small RNAs in animals. Nat Rev Mol Cell Biol 10: 126-139. doi:10.1038/nrm2632

Kim Y-K, Kim B, Kim VN. 2016. Re-evaluation of the roles of DROSHA, Exportin 5, and DICER in microRNA biogenesis. Proc Natl Acad Sci 113: E1881-E1889. doi:10.1073/pnas.1602532113 
Kozomara A, Griffiths-Jones S. 2014. miRBase: annotating high confidence microRNAs using deep sequencing data. Nucleic Acids Res 42: D68-D73. doi:10.1093/nar/gkt1181

Langmead B, Salzberg SL. 2012. Fast gapped-read alignment with Bowtie 2. Nat Methods 9: 357. doi:10.1038/nmeth.1923

Lorenz R, Bernhart SH, Höner zu Siederdissen C, Tafer H, Flamm C, Stadler PF, Hofacker IL. 2011. ViennaRNA Package 2.0. Algorithms Mol Biol 6: 26. doi:10.1186/1748-7188-6-26

Martin M. 2011. Cutadapt removes adapter sequences from highthroughput sequencing reads. EMBnet.journal 17: 10-12. doi:10 .14806/ej.17.1.200

Meijer HA, Smith EM, Bushell M. 2014. Regulation of miRNA strand selection: follow the leader? Biochem Soc Trans 42: 1135-1140. doi:10.1042/BST20140142

Mencía A, Modamio-Høybjør S, Redshaw N, Morín M, MayoMerino F, Olavarrieta L, Aguirre LA, del Castillo I, Steel KP, Dalmay T, et al. 2009. Mutations in the seed region of human miR-96 are responsible for nonsyndromic progressive hearing loss. Nat Genet 41: 609-613. doi:10.1038/ng.355

Mitchell PS, Parkin RK, Kroh EM, Fritz BR, Wyman SK, PogosovaAgadjanyan EL, Peterson A, Noteboom J, O'Briant KC, Allen A, et al. 2008. Circulating microRNAs as stable blood-based markers for cancer detection. Proc Natl Acad Sci 105: 10513-10518. doi:10.1073/pnas.0804549105

Morales S, Monzo M, Navarro A. 2017. Epigenetic regulation mechanisms of microRNA expression. Biomol Concepts 8: 203-212. doi:10.1515/bmc-2017-0024

Neilsen CT, Goodall GJ, Bracken CP. 2012. IsomiRs-the overlooked repertoire in the dynamic microRNAome. Trends Genet 28: 544 549. doi:10.1016/j.tig.2012.07.005

Nishikura K. 2016. A-to-l editing of coding and non-coding RNAs by ADARs. Nat Rev Mol Cell Biol 17: 83-96. doi:10.1038/nrm.2015.4

Oudot-Mellakh T, Cohen W, Germain M, Saut N, Kallel C, Zelenika D, Lathrop M, Trégouët D-A, Morange P-E. 2012. Genome wide association study for plasma levels of natural anticoagulant inhibitors and protein $\mathrm{C}$ anticoagulant pathway: the MARTHA project. $\mathrm{Br} \mathrm{J}$ Haematol 157: 230-239. doi:10.1111/j.1365-2141.2011.09025.x

Pulcrano-Nicolas A-S, Proust C, Clarençon F, Jacquens A, Perret C, Roux M, Shotar E, Thibord F, Puybasset L, Garnier S, et al. 2018. Whole-blood miRNA sequencing profiling for vasospasm in patients with aneurysmal subarachnoid hemorrhage. Stroke 49: 2220-2223. doi:10.1161/STROKEAHA.118.021101
Roux M, Perret C, Feigerlova E, Mohand Oumoussa B, Saulnier P-J, Proust C, Trégouët D-A, Hadjadj S. 2018. Plasma levels of hsamiR-152-3p are associated with diabetic nephropathy in patients with type 2 diabetes. Nephrol Dial Transplant 33: 2201-2207. doi:10.1093/ndt/gfx367

Russell PH, Vestal B, Shi W, Rudra PD, Dowell R, Radcliffe R, Saba L, Kechris K. 2018. miR-MaGiC improves quantification accuracy for small RNA-seq. BMC Res Notes 11: 296. doi:10.1186/ s13104-018-3418-2

Tam S, de Borja R, Tsao M-S, McPherson JD. 2014. Robust global microRNA expression profiling using next-generation sequencing technologies. Lab Invest 94: 350-358. doi:10.1038/labinvest .2013 .157

Urgese G, Paciello G, Acquaviva A, Ficarra E. 2016. isomiR-SEA: an RNA-seq analysis tool for miRNAs/isomiRs expression level profiling and miRNA-mRNA interaction sites evaluation. BMC Bioinformatics 17: 148. doi:10.1186/s12859-016-0958-0

Wallaert A, Van Loocke W, Hernandez L, Taghon T, Speleman F, Van Vlierberghe P. 2017. Comprehensive miRNA expression profiling in human T-cell acute lymphoblastic leukemia by small RNA-sequencing. Sci Rep 7: 7901. doi:10.1038/s41598-017-08148-x

Wright C, Rajpurohit A, Burke EE, Williams C, Collado-Torres L, Kimos M, Brandon NJ, Cross AJ, Jaffe AE, Weinberger DR, et al. 2019. Comprehensive assessment of multiple biases in small RNA sequencing reveals significant differences in the performance of widely used methods. bioRxiv doi: 10.1101/445437

Wu X, Kim T-K, Baxter D, Scherler K, Gordon A, Fong O, Etheridge A, Galas DJ, Wang K. 2017. sRNAnalyzer-a flexible and customizable small RNA sequencing data analysis pipeline. Nucleic Acids Res 45: 12140-12151. doi:10.1093/nar/gkx999

Wu CW, Evans JM, Huang S, Mahoney DW, Dukek BA, Taylor WR, Yab TC, Smyrk TC, Jen J, Kisiel JB, et al. 2018. A Comprehensive Approach to Sequence-oriented IsomiR annotation (CASMIR): demonstration with IsomiR profiling in colorectal neoplasia. BMC Genomics 19: 401. doi:10.1186/s12864-018-4794-7

Wyman SK, Knouf EC, Parkin RK, Fritz BR, Lin DW, Dennis LM, Krouse MA, Webster PJ, Tewari M. 2011. Post-transcriptional generation of miRNA variants by multiple nucleotidyl transferases contributes to miRNA transcriptome complexity. Genome Res 21: 1450-1461. doi:10.1101/gr.118059.110

Ziemann M, Kaspi A, El-Osta A. 2016. Evaluation of microRNA alignment techniques. RNA 22: 1120-1138. doi:10.1261/rna.055509 .115 

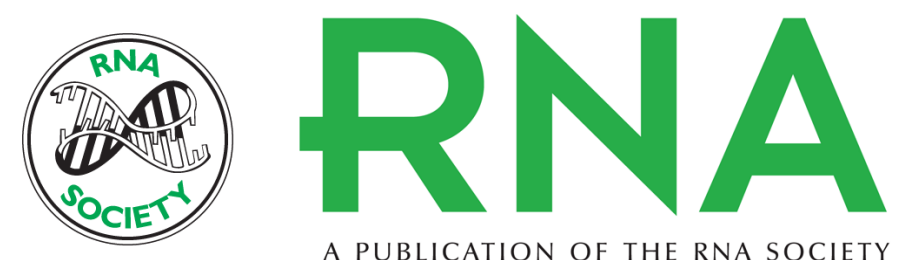

A PUBLICATION OF THE RNA SOCIETY

\section{OPTIMIR, a novel algorithm for integrating available genome-wide genotype data into miRNA sequence alignment analysis}

Florian Thibord, Claire Perret, Maguelonne Roux, et al.

RNA 2019 25: 657-668 originally published online February 28, 2019

Access the most recent version at doi:10.1261/rna.069708.118

\section{Supplemental http://rnajournal.cshlp.org/content/suppl/2019/02/28/rna.069708.118.DC1 \\ Material}

References This article cites 46 articles, 11 of which can be accessed free at: http://rnajournal.cshlp.org/content/25/6/657.full.html\#ref-list-1

Open Access Freely available online through the RNA Open Access option.

Creative This article, published in $R N A$, is available under a Creative Commons License Commons (Attribution 4.0 International), as described at

License http://creativecommons.org/licenses/by/4.0/.

Email Alerting Receive free email alerts when new articles cite this article - sign up in the box at the Service top right corner of the article or click here.

\section{|||||||| Providing Precise Solutions for} your research.

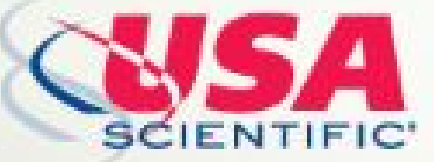

To subscribe to $R N A$ go to:

http://rnajournal.cshlp.org/subscriptions 